\title{
Proposal and Evaluation of 8-ary Elliptical Phase Shift Keying
}

\author{
Chunyi Song, Shigeru Shimamoto \\ Graduate School of Global Information and Telecommunication Studies, Waseda University \\ Waseda Bldg. 29-7, 1-3-10 Nishi-Waseda, Shinjuku-Ku, Tokyo 169-0051, Japan \\ song@sl.giti.waseda.ac.jp shima@giti.waseda.ac.jp
}

\begin{abstract}
Some modulation schemes with the general name of Elliptical Modulation Schemes, have been proposed and generally evaluated through computer simulations in our previous works, namely are Eccentricity Shift Keying (ESK), Inclination Angle Shift Keying (IASK) and Elliptical Phase Shift Keying (EPSK). This paper aimed to propose and evaluate 8-ary Elliptical Phase Shift Keying (8-EPSK), a new modulation scheme with 3-bit information transmission capability. In the paper, 8-EPSK was defined mathematically at first; it was then evaluated mathematically and by computer simulation in terms of BER, along with comparison to 8PSK. Both analysis results and simulation results showed that 8-EPSK outperforms 8PSK.
\end{abstract}

Keywords - Modulation; 8-EPSK; 8PSK; BER

\section{INTRODUCTION}

In addition to amplitude, frequency and phase, of a sinusoid signal used in existing modulation schemes, our proposed Elliptical Modulation Schemes, introduce additional attributes such as eccentricity, offset inclination angle, rotation frequency and rotation direction of an elliptical signal, through employing geometrical characteristics of an ellipse to manipulate a signal[1]. Introduction of new modulation variables provides Elliptical Modulation Schemes with higher flexibility, which implies that considerable types of efficient modulation schemes can be developed.

Some modulation schemes with the general name of Elliptical Modulation Schemes have been proposed and generally evaluated through computer simulations in our previous works [1], [2], namely are Eccentricity Shift Keying (ESK), Rotation Frequency Shift Keying (RFSK), Inclination Angle Shift Keying (IASK) and Elliptical Phase Shift Keying (EPSK). As an instance of EPSK, 8-ary Elliptical Phase Shift Keying (8-EPSK) has been referred and generally evaluated through computer simulations [2]. In our previous works, mathematical analysis on definitions, signal characteristics and error probability, haven't been given out. Beyond these, configurations of receiver also need to be discussed from point view of implementation complexity.

In this paper, 8-EPSK will be defined in section II, it will then be analysed mathematically in terms of BER in section III. To give an impression on implementation complexity, receiver configuration of 8-EPSK will be illustrated in section IV. Finally, simulation results on BER performance will be analysed and conclusions will be drawn in section $\mathrm{V}$.

\section{DEFINITIONS OF 8-EPSK}

As one of most basic Elliptical Modulation Schemes, at first definition of IASK will be reviewed; then definitions of 8-EPSK will be derived from EPSK mathematically, with general expressions of its signals and followed by general discussion on two types of constellation diagrams.

\section{A. Inclination Angle Shift Keying (IASK)}

Distinctive waveforms can be produced by changing offset inclination angles, which suggests that binary numbers can be represented by ellipses inclined at different angles. This is defined as IASK. As an instance, two ellipses showed in Fig. 1 can be used to represent binary digit of 0 and 1 respectively.

IASK signals are expressed as:

$$
s(t)=a \sqrt{\frac{1-e_{c}{ }^{2}}{1-e_{c}{ }^{2} \cos ^{2}\left(w_{r} t-\alpha_{i}\right)}} \cos w_{r} t,
$$

where $a=$ semi-major axis, $\omega_{r}=$ revolution angular frequency, $e_{c}=$ eccentricity, $\alpha_{i}=$ offset inclination angle.

\section{B. General Expression of EPSK Signals}

In Elliptical Modulation Schemes, EPSK has been defined through combining effect of offset inclination angle and that of signal's phase. EPSK signals can be generally expressed as:

$$
s(t)=a \sqrt{\frac{1-e_{c}^{2}}{1-e_{c}^{2} \cos ^{2}\left(w_{r} t+\phi_{j}-\alpha_{i}\right)}} \cos \left(w_{r} t+\phi_{j}\right),
$$

where $a, e_{c}, \omega_{1}$ and $\alpha_{i}$ are the same with those in (1); $\phi_{j}=$ signal's phase; $\alpha_{i}$ and $\phi_{j}$ are supposed to have $N$ and $K$ discrete values respectively $(N \geq 1, K \geq 2)$.

\section{8-ary Elliptical Phase Shift Keying}

By fixing $N$ to value of " 2 " and $M$ to value of " 4 " in (2), 8 -EPSK can be defined through combining QPSK and IASK. In 8-EPSK, "2" offset inclination angles are used to represent the first binary bits of 0 and 1, " 4 " phases at four quadrants in each

This research project is supported by Japanese Ministry of Science and Education Grants-in-Aid for Scientific Research, Exploratory Research NO.15656094, and by Waseda University Special Grant. 
ellipse are then assigned with 2-bit message sequences from $00 \sim 11$. Thus, 8-EPSK is capable of 3-bit information transmission under the same carrier frequency.

Without losing a generality, in our definitions, two offset inclination angles are set to be $\pi / 4$ and $-\pi / 4$, and four phases are set to be $\pi / 4,3 \pi / 4,5 \pi / 4$ and $7 \pi / 4$, respectively. Substituting all eight pairs of $\alpha_{i}$ and $\phi_{j}$ to (2), and then simplifying expression of each unit signal, general expressions of 8-EPSK signals can be achieved as:

$$
s(t)=a \sqrt{\frac{1-e_{c}^{2}}{1-e_{c}^{2} \cos ^{2}\left(w_{r} t+j \cdot \pi / 2\right)}} \cos \left[w_{r} t+(1+2 i) \cdot \pi / 4\right],
$$

where $i=0,1,2,3 ; j=0,1$; the way of deciding value of $j$ will be discussed later. Waveforms of 8-EPSK signals then can be made in Fig.2.

According to the way of assigning message sequences to phases in each ellipse, two types of Constellation diagrams can be defined for 8-EPSK as showed in (a) and (b) of Fig.3. In (a), 2-bit difference lies in neighboring signals, and radius of decision region $r_{001-010}=\sqrt{2} b / 2$; while in (b), 2-bit difference lies in each pair of diametrically opposite signals, and $r_{001-010}$ $=b$. Beyond this, two constellation points with the maximum distance has been assigned to signals with 2-bit difference in (b), instead of signals with 1-bit difference in (a). This also illustrates

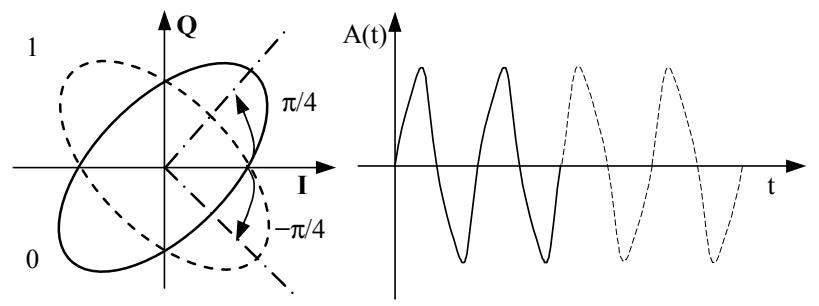

Figure 1. An example of IASK signals

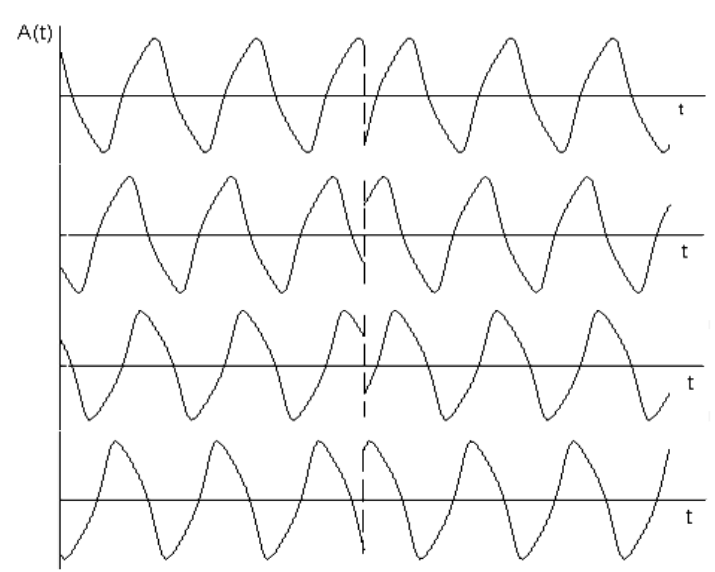

Figure 2. Waveforms of 8-EPSK signals, where $A(t)$ is Amplitude. Upper two rows are signals in ellipse inclined at $\pi / 4$, while lower two are signals in ellipse inclined at $-\pi / 4$. Each row includes two signals with phase-shift of " $\pi$."

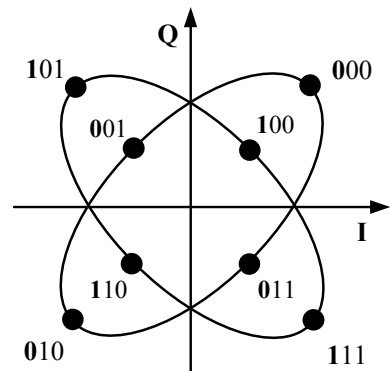

(a)

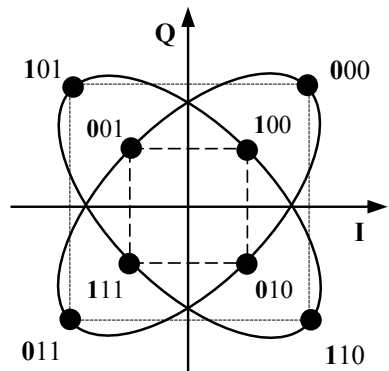

(b)
Figure 3. Constellation diagrams of 8-EPSK

that assignment way in (b) is more efficient than that in (a). Thus, (b) has been selected as Constellation diagrams of 8EPSK.

8-EPSK is defined through combining IASK and phase modulation. Either graphically from Fig.3, or mathematically from (3), it can be seen that envelope of 8-EPSK signal keep changing in one symbol duration. Quadrature Amplitude Modulation (QAM) may be viewed as form of combined digital-amplitude modulation and digital-phase modulation. Although QAM is not envelop-constant with respect to different signals, its envelop keeps constant for each unit signal in one symbol duration. Based on this analysis of their difference, BER performance of 8-EPSK will not compared with that of QAM, but with that of 8PSK.

\section{MATHEMATICAL ANALYSIS ON ERROR PROBABILITY}

\section{A. Euclidean Distance of 8-EPSK}

Mathematically, degree of similarity between two signals is expressed in terms of their correlation coefficient [3]:

$$
\rho=\frac{\int_{T} s_{1}(t) \cdot s_{2}(t) d t}{\int_{T} s_{1}^{2}(t) d t},
$$

or in terms of their Euclidean distance:

$$
\begin{aligned}
D^{2} & =\int_{T}\left[s_{1}(t)-s_{2}(t)\right]^{2} d t \\
& =E_{1}+E_{2}-2 \sqrt{E_{1} \cdot E_{2}} \cdot \rho,
\end{aligned}
$$

where $\mathrm{E}$ is the mean energy over one symbol duration. When $\mathrm{E}_{1}=\mathrm{E}_{2},(5)$ can be simplified to:

$$
D^{2}=2 \cdot E_{b i t} \cdot(1-\rho)
$$

By using (4), correlation coefficient of signals " 100 " and " 110 " can be expressed as:

$$
\rho=\frac{\int_{T} a^{2} \frac{1-e_{c}{ }^{2}}{1-e_{c}^{2} \cos ^{2} w_{r} t} \cos \left(w_{r} t+\pi / 4\right) \cos \left(w_{r} t+7 \pi / 4\right) d t}{\int_{T} a^{2} \frac{1-e_{c}^{2}}{1-e_{c}{ }^{2} \cos ^{2} w_{r} t} \cos ^{2}\left(w_{r} t+\pi / 4\right) d t} .
$$


For the difficulty in achieving numeric value of (7) through algebric computation, approximate computation is used through calculating values of 1 million sampling points in one symbol duration. The obtained value is positive. From Fig. 2 and Fig.3, it can be found that beyond phase shift of $\pi / 2$ between signals " 100 " and " 110 ", waveform of " 100 " leans to the right, while waveform of " 110 " leans to the left. We think that correlation coefficient of these two signals should be negative, and thus conclude that (4) is not an accurate way to measure similarity degree of two elliptical signals.

From Fig.4, Euclidean distance need to be discussed include:

1) Euclidean distance between neighboring signals in the same ellipse. The only difference of such two signals is phase shift of $\pi / 2$, and the correlation coefficient is 0 . Thus, the Euclidean distance can be achieved from (6) as:

$$
D=\sqrt{2 \cdot E_{b i t} \cdot(1-\rho)}=\sqrt{2} \sqrt{E},
$$

which is the same with minimum Euclidean distance of QPSK.

2) Euclidean distance between signals with the same phase but lie in different ellipses. As we analyzed above, correlation coefficient of elliptical signals achieved by using (4) might be magnified. Here, two signals have the same phase, and they can be regarded as a pair of IASK signals. Based on what have been achieved on BER performance of IASK in our previous works [2], we think that correlation coefficient smaller than $\sqrt{2} / 2$ can be obtained by setting eccentricity to be some certain values. As we know, Correlation coefficient of two neighboring 8PSK signals equals to $\sqrt{2} / 2$. From (6), Euclidean distance is inversely proportional to correlation coefficient, so the Euclidean distance discussed here can be larger than the minimum Euclidean distance of 8PSK.

Based on above analysis, we conclude that 8-EPSK can have larger minimum Euclidean distance than 8PSK, by setting eccentricity to be some certain values.

\section{B. Decision Region and BER Performance of 8-EPSK}

Distance between constellation points at the same quadrant but in different ellipses, such as $d_{110-010}$, can not be simply expressed by their geometrical distance, and here, the distance is measured by taking its Euclidean distance as reference. As we concluded above, Euclidean distance of signals "010" and " 110 " is larger than 8PSK's minimum Euclidean distance, at some certain values of eccentricity. The same conclusion then can be drawn with respect to the distance between constellation points. In 8-EPSK, this distance is directly proportional to the value of eccentricity, thus it is generally called " $d_{e c}$ " in the following discussions.

From Fig.4, Decision region of 8-EPSK is divided by two axes, and radii of decision region for different signals are different, which can be generally expressed as:

$$
r_{010 \rightarrow 001}=r_{001 \rightarrow 010}=b,
$$

$$
\begin{aligned}
& r_{010 \rightarrow 011}=\sqrt{2} b / 2, \\
& r_{011 \rightarrow 010}=\sqrt{2} a / 2, \\
& b=\sqrt{a^{2}-a^{2} e_{c}^{2}},
\end{aligned}
$$

where $a=$ semi-major axis, $e_{c}=$ eccentricity; $a$ is directly proportional to $e_{c}$. Substituting (12) to (9) and (10), we have:

$$
\begin{gathered}
r_{010 \rightarrow 001}=r_{001 \rightarrow 010}=a \sqrt{1-e_{c}^{2}}, \\
r_{010 \rightarrow 011}=a / 2 \sqrt{2-2 e_{c}^{2}} .
\end{gathered}
$$

Known graphically from Fig.4, (13) and (14) are inversely proportional to the eccentricity, while (11) is directly proportional to the eccentricity. Among these three (13) is the only one stands for 2-bit difference, and (11) is larger than (14) at any eccentricities. To achieve the best performance, we should try to average (11), (14) and " $d_{e c}$ ", and make (13) to be the largest by setting eccentricity to be some small values. Without considering " $d_{e c}$ ", better performance can be achieved just through decreasing value of eccentricity. As $d_{e c}$ is directly proportional to the eccentricity, the best performance can be achieved when $d_{e c}$ is twice of (14):

$$
d_{e c}=2 \times r_{010 \rightarrow 011}
$$

Eccentricity obtained from (15) is called optimum eccentricity. Defining the optimum eccentricity as " $k$ ", then the minimum radius of decision region can be expressed as:

$$
r_{\min }=r_{e c=k}=\frac{a}{2} \sqrt{2-2 k^{2}}
$$

Defining distance from constellation point to the centre point of constellation diagrams in 8PSK as "A" (Fig.4), then some values of $a$ corresponding to typical $e_{c}$, and value of (16), can be obtained and summarized in Table I.

Characteristic on effect of eccentricity to performance of IASK has been investigated in [2], from the result it can be concluded that " $k$ "in (16) should be larger than 0.4 . From Table I, the minimum radius of decision region has the smallest value when

$$
r_{\min }=r_{e c=0.9}=0.47 \mathrm{~A} \text {. }
$$
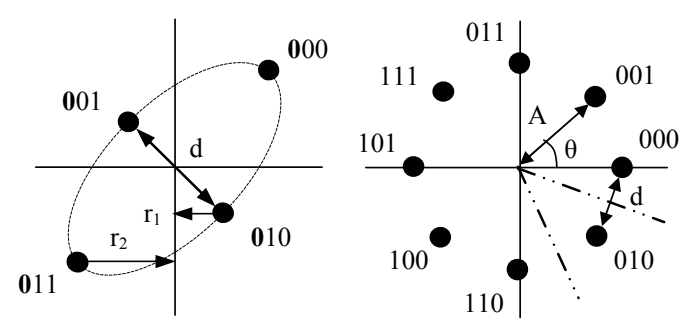

Figure 4. Signal space of 8-EPSK and 8PSK 
TABLE I. CORRESPONDING VALUES OF SEMI-MAJOR AXIS AND MINIMUM RADII OF DECISION REGION TO SOME TYPICAL ECCENTRICITY

\begin{tabular}{|c|c|c|c|c|c|c|}
\hline$e_{c}$ & 0.9 & 0.8 & 0.7 & 0.6 & 0.5 & 0.4 \\
\hline$a$ & $1.51 \mathrm{~A}$ & $1.29 \mathrm{~A}$ & $1.18 \mathrm{~A}$ & $1.11 \mathrm{~A}$ & $1.07 \mathrm{~A}$ & $1.04 \mathrm{~A}$ \\
\hline$r_{\min }$ & $0.47 \mathrm{~A}$ & $0.55 \mathrm{~A}$ & $0.59 \mathrm{~A}$ & $0.63 \mathrm{~A}$ & $0.65 \mathrm{~A}$ & $0.67 \mathrm{~A}$ \\
\hline
\end{tabular}

From Fig.4, the minimum radius of decision region in 8PSK is:

$$
r_{\min }=A \sin (\theta / 2)=A \sin (\pi / 8) \approx 0.38 A .
$$

From (17) and (18), 8-EPSK has larger minimum radius of decision region than 8 PSK.

Theoretic BER of $M$-ary PSK is given our as:

$$
P_{e}=2 Q\left(\frac{d}{2 \sigma}\right),
$$

where $\mathrm{d}$ is the minimum distance between constellation points [4], which is twice of minimum radius of decision region.

Doubling (17) and (18), and then substituting the achieved results to (19), we have:

$$
r_{8-E P S K}>r_{8 P S K} \Leftrightarrow d_{8-E P S K}>d \Leftrightarrow P_{e_{-} 8-E P S K}<P_{e_{-} 8 P S K}
$$

For 8-EPSK, all signals have the same possibility to be transmitted, thus accurate $d_{8 \text {-EPSK }}$ should be the average value and the performance in (20) will be improved. Based on above analysis, we conclude that 8-EPSK outperforms 8PSK on error performance.

\section{RECEIVER OF 8-EPSK}

Demodulation of 8-EPSK signals is consisted of two steps: the last two bits can be determined by identifying which quadrant the coming signal lies in; and then the first bit can be detected by identifying which ellipse has been used to send out the information. Thus, we combined coherent demodulator and correlation detector in the receiver (Fig.5) [5] [7].

Last two bits of received signal can be detected by determine its phase, and the detection can be accomplished by using QPSK receiver block diagram directly. However, the carrier recovery circuit in Fig.5 is more complex in comparison with that in QPSK receiver. From (3), beyond carrier frequency, value of " $j$ " also needs to be determined when carrying out carrier recovery. In Fig.3, four signals consists of small-size square have odd number of " 1 ", and " $j$ " in (3) equals to 1 ; four signals consists of big-size square have even number of " 1 ", and then " $j$ " in (3) equals to 0 . This method of determining value of " $j$ " can be employed in modulation, and then carrier recovery circuit in the receiver can obtain the information from modulator.

Received signal lies in either of four quadrants. Detection of each pair of signals in the same quadrant demands one correlation detector, which means four correlation detectors are needed in the receiver. Note that for both cosine and sine, effect of phase-shift of $\pi$ equals to effect of multiplying "- 1 ," and they have the same squared values as:

$$
\begin{aligned}
& \cos \left(\omega_{r} t+\pi\right)=-\cos \left(\omega_{r} t\right) \Rightarrow\left[\cos \left(\omega_{r} t+\pi\right)\right]^{2}=\left[\cos \left(\omega_{r} t\right)\right]^{2}, \\
& \sin \left(\omega_{r} t+\pi\right)=-\sin \left(\omega_{r} t\right) \Rightarrow\left[\sin \left(\omega_{r} t+\pi\right)\right]^{2}=\left[\sin \left(\omega_{r} t\right)\right]^{2} .
\end{aligned}
$$

Thus, in Fig.6, through adding a squaring operation block following the product integrator, two signals with phase-shift of $\pi$ can be detected by using the same reference signals. Based on this analysis, we reduced the number of correlation detectors from four to two in simulations. Without losing generality, signals obtained from (3) by setting $(i, \mathrm{j})$ to be $(0,0),(0,1),(1$, $0)$ and $(1,1)$, are used as reference signals. They are expressed as $S_{\text {ref_1 }}, S_{\text {ref_ } 2}, S_{\text {ref_3 }}$ and $S_{\text {ref } 44}$ in order.

In Fig.5, switch "w" is used to select one from two correlation detectors. If the received signal is identified in the first or the third quadrant, "correlation detector1" will be connected and activated, which is using reference signals $S_{\text {ref } 1}$ and $S_{\text {ref } 3}$; if the detected data lie in the second or the fourth quadrant, "correlation detector2" will then be activated, and the reference signals are consisted of $S_{r e f_{-} 2}$ and $S_{r e f} 4$.

In Fig.6, the upper two channels are configured to detect signals in ellipse inclined at $\pi / 4$, while the lower two branches are configured to detect signals in ellipse inclined at $-\pi / 4$ [8].

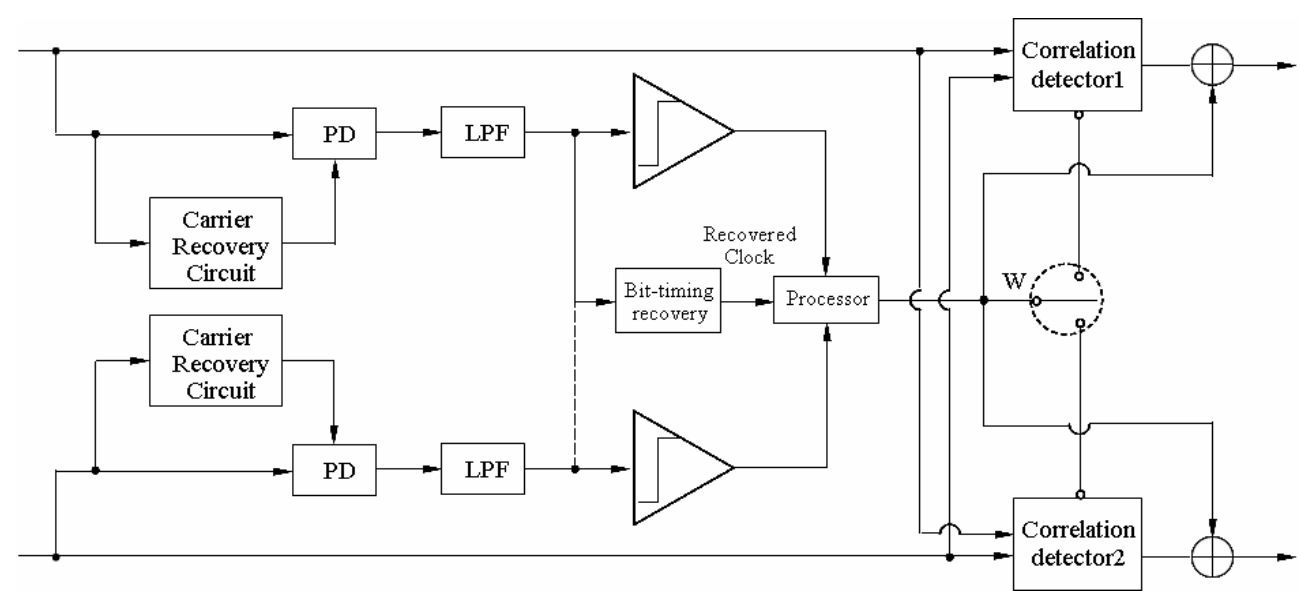

Figure 5. 8-EPSK Receiver block diagram 


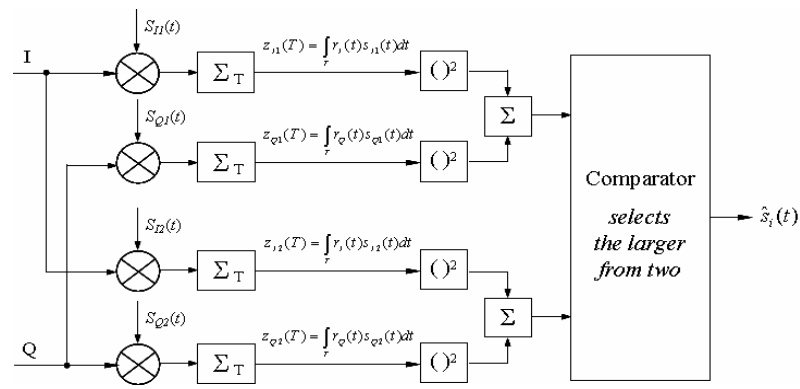

Figure 6. Modified correlation detector of 8-EPSK

\section{Simulation Results AND CONCLUSIONS}

In simulations, BER performance of 8-EPSK is investigated under AWGN and in Rayleigh fading channel respectively [9]. To investigate the effect of eccentricity to the error performance, eccentricity is set to be a variable from 0.1 to 0.9 , with unit increase of 0.1 .

Simulation results in Fig. 7 and Fig. 8 showed that either under AWGN or in Rayleigh fading channel, 8-EPSK has better performance than 8PSK when eccentricity is set to be larger than 0.5 . This provides 8 -EPSK with high flexibility to make trade-off on error performance and bandwidth efficiency in implementations, by setting eccentricity to optimum value.

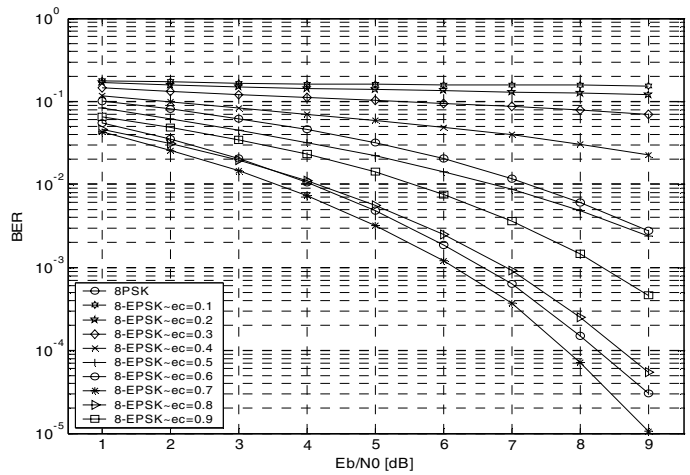

Figure 7. BER performance comparison among 8PSK and 8-EPSK at difference values of eccentricity, under AWGN

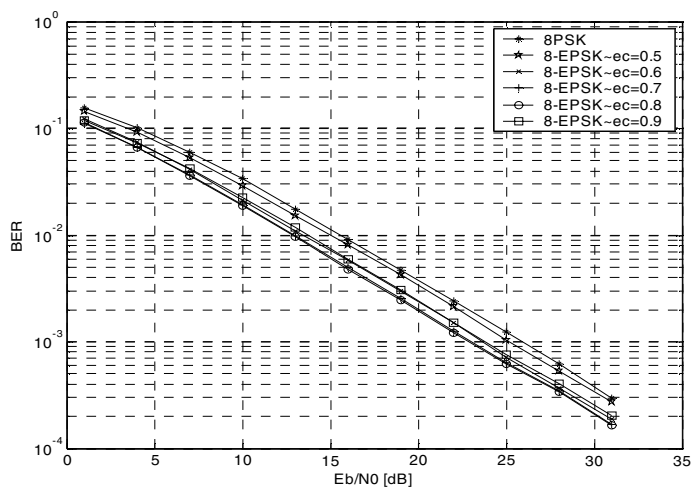

Figure 8. BER performance comparison among 8PSK and 8-EPSK at difference values of eccentricity, in Rayleigh fading channel
From simulation results, it can also be found that in AWGN channel, the best performance is achieved at eccentricity of 0.7 , and 8-EPSK at eccentricity of 0.6 outperforms 8-EPSK at eccentricity of 0.8 and 0.9 (Fig.7); while in Rayleigh fading channel, 8-EPSK at eccentricities of 0.7 and 0.8 have undistinguishable performance, 8-EPSK at eccentricities of 0.6 and 0.9 also have undistinguishable performance (Fig.8). After adding channel fading to AWGN, the optimum eccentricity area has shifted to larger values.

According to analysis in section III and section IV, increase of eccentricity can improve the performance of correlation detection, at the expense of decreasing minimum radius of decision region. So the up-shift of optimum eccentricity can be illustrated that, performance of correlation detection is more vulnerable to the worsening of communication environment, in comparison with that of phase-detection used here. From point view of mathematical analysis, (16) can only be existed at the larger value of eccentricity after adding channel fading to AWGN. This also implies that some new detection methods more efficient than correlation detection might be developed, which should be capable of making better use of distinction between two signals in different ellipses.

Since Correlation Coefficient can not express degree of similarity between two elliptical signals accurately, new mathematical method needs to be developed in the future research. Beyond this, pulse-shaping filter based on elliptical signals is necessary in evaluating spectral efficiency of 8 -EPSK, which is also part of our main works in the future.

\section{ACKNOWLEDGMENT}

Authors thank The Telecommunications Advancement Foundation of Japan, and Denki-Densi-Jyoho-Gakujyutsusinkou Foundation of Japan, for their financial support.

\section{REFERENCES}

[1] S.Shimamoto, "Proposal of new modulation scheme employing elliptical circle." IEEE Wireless Communications and Networking Conference 2003. March 2003, New Orleans, USA.

[2] Chunyi Song and S.Shimamoto, "Evaluations of Elliptical Modulation Scheme." IEEE Wireless Communications and Networking Conference 2004. March 2004, Atlanta, Georgia USA.

[3] B.P.Lathi, "Signal Comparison: Correlation" in Modern Digital and Analog Communication System, Oxford University Press, Oxford, 1998, pp35-pp40

[4] Alister Burr, "Basic Linear Modulation Schemes," in Modulation and Coding for Wireless Communications, Prentice Hall, 2001, pp36-pp39.

[5] Paul H. Young, "QPSK Demodulation," in Electronic Communication Techniques (Fourth Edition), Prentice Hall, 1999, pp703-pp708.

[6] S.Rappaport, "Binary Phase Shift Keying" in Wireless CommunicationsPrinciples and Practice (Second Edition), Prentice Hall, 2002, pp295-pp298.

[7] Alister Burr, "Modem Design" in Modulation and Coding for Wireless Communications, Prentice Hall, 2001, pp77-pp109.

[8] B.Sklar, "Correlation Receiver," in Digital CommunicationsFundamentals and Applications ( Second Edition ), Prentice Hall: New Jersey, 2001, pp.178-183.

[9] Hiroshi Harada and Ramjee Prasad, "Definition of a Radio Communication Channel," in Simulation and Software Radio, London: Artech House, 2002,.pp50-pp61. 